Vol. 67, N. ${ }^{\circ}$ Io6 (noviembre 2015), I2I-I43

\title{
SISTEMAS DE PROTECCIÓN SOCIAL PARA ADULTOS MAYORES EN AMÉRICA LATINA: AGENDA PENDIENTE EN EL SISTEMA DE PENSIONES EN ECUADOR
}

\author{
JEANNETTE SÁNCHEZ \\ Universidad Central del Ecuador
}

Recepción manuscrito: 4 de agosto de 2015

Aceptación versión final: 30 de octubre de 2015

\begin{abstract}
RESUMEN En este trabajo se reflexiona sobre el sistema de pensiones jubilares como parte importante del sistema de protección social para la población de adultos mayores en América Latina y particularmente en Ecuador. A partir de los avances y límites del desempeño social se proponen algunos criterios mínimos para una reforma integral a la seguridad social con visión universal para el caso de Ecuador. Se destaca la necesidad de una estrategia basada en acciones urgentes y estructurales, concentrar los subsidios en la población de menos recursos excluida del sistema de seguridad social, complementar acciones con la política de salud a fin de promover un envejecimiento en mejores condiciones, y enfrentar los problemas estructurales del mercado laboral.

PALABRAS CLAVE Economía demográfica y laboral, economía de adultos mayores, políticas de jubilación, trabajo informal, bienestar y pobreza.
\end{abstract}

ABSTRACT In this work, we reflect on the retirement pension plan as an important part of the social protection system for elderly population in Latin America, and, particularly, in Ecuador. Based on the progress and limits of social performance we propose some minimum criteria for a comprehensive reform in social security with a universal vision in Ecuador's case. We highlight the need for a strategy based on urgent structural action, focusing the subsidies on the population with the least resources, who is excluded from the social security system, complementing actions with health politics in order to promote growing old in the best conditions, and facing the work market structural problems.

KEYWORDS Labor and demographic economics, elderly people's economics, retirement politics, informal work, welfare, well-being and poverty.

JEL CODES J14, J26, J46, I3O.

\section{INTRODUCCIÓN}

El presente trabajo - que es una versión ajustada de la ponencia «Sistemas de protección social para adultos mayores en América Latina: agenda pendiente desde la experiencia ecuatoriana» presentada en el v Congreso de la Red Española de Política Social (REPs) titulado 
«Desigualdad y democracia: políticas públicas e innovación social» en Barcelona, España, el 5 y 6 de febrero de 2015- analiza los sistemas de protección social para la población adulta mayor en América Latina y Ecuador, particularmente en lo que se refiere a los sistemas de pensiones. En el contexto de los avances y desafíos del desempeño y las políticas sociales de la región y de Ecuador se proponen criterios para la construcción de una agenda aplicable a Ecuador, y a países en desarrollo con características demográficas y socioeconómicas parecidas a las ecuatorianas. A diferencia del caso europeo, en América Latina existen Estados de bienestar más débiles, una gran variedad de políticas sociales que marcan desempeños distintos y diferentes alcances de universalidades enfocadas hacia servicios clave como la educación, la salud y la protección social, lo que, en conjunto con mercados laborales heterogéneos, reproduce la desigualdad. Los sistemas de pensiones presentan déficits importantes para apoyar a una población de edad avanzada que crece con rapidez y que, casi siempre, excluyen a la población más pobre.

Los problemas estructurales que se evidencian en mercados laborales con alta informalidad y sectores de baja productividad que no logran capturar un empleo digno, están detrás de la baja cobertura de la seguridad social y las restricciones en los sistemas de protección social. Si bien se han dado avances importantes en la política y desempeño social, quedan algunos cuellos de botella importantes para implementar sistemas universales de protección social, particularmente en los países de menor desarrollo relativo dentro de la región como es el caso ecuatoriano. Las políticas para la población en edad avanzada han sido las políticas menos desarrolladas, pues deben enfrentar grandes desafíos, tanto en recursos como en estrategias e instrumentos para promover un envejecimiento digno. El peor escenario lo enfrenta la población adulta mayor que no tiene pensión jubilar y que se encuentra en situación de pobreza, o que, desprovista de un seguro público o privado, se encuentra vulnerable ante una enfermedad o contingencia que le lleve a un estado de pobreza.

La región, por otra parte, experimenta un heterogéneo proceso de envejecimiento de su población. Ecuador, con una población mayoritariamente joven, integra el grupo de países con menor envejecimiento relativo respecto al promedio regional, ventana de oportunidad que sin embargo durará poco tiempo, por lo cual es fundamental pensar en una propuesta que permita estructurar y poner en funcionamiento sistemas de protección social adecuados y sostenibles en el tiempo en el cual las pensiones jubilares juegan un rol muy importante. En un contexto de desaceleración como el que actualmente vive la región, que afecta particularmente a la población adulta mayor - sobre todo aquella que cuenta con menos posibilidades de acceder a ingresos regulares - es preocupación común crear y consolidar un sistema de protección social para enfrentar riesgos y contingencias para los cuales ni las familias, ni la comunidad, ni el Estado se encuentran preparados.

Este trabajo parte de un breve análisis de la situación demográfica, el envejecimiento y la seguridad social en la región y Ecuador. En segundo lugar, analiza el contexto del desempeño social y los desafíos de la coyuntura económica. En tercer lugar, plantea el estado de arte de las políticas de protección social, particularmente del sistema de seguridad social y de las transferencias monetarias, para revelar los déficits en el sistema de pensiones para la vejez. En cuarto lugar, presenta el caso ecuatoriano. Finalmente, establece algunas conclusiones y algunos 
Tabla 1. Índice de envejecimiento en América Latina

\begin{tabular}{lc}
\hline AÑO & ÍNDICE DE ENVEJECIMIENTO EN AMÉRICA LATINA \\
\hline 1950 & 13,9 \\
1970 & 14,7 \\
1990 & 19,9 \\
2000 & 25,3 \\
2010 & 34,7 \\
2015 & 42,8 \\
\hline
\end{tabular}

Fuente: CEPAL (2014). Observatorio demográfico 2013. Nota: Índice de envejecimiento: número de población de 65 años por cada 100 personas menores de 15 años.

Figura 1. Afilicación a sistemas de pensiones y de salud entre los asalariados de 15 años y más en América Latina

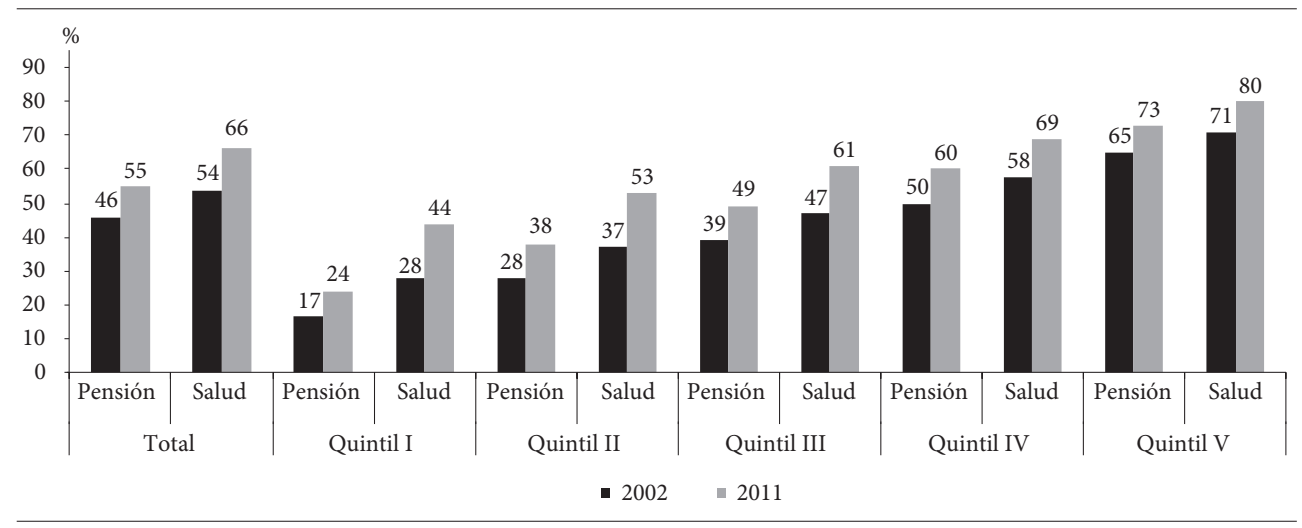

Fuente: CEPAL (2013).

criterios para la construcción de un sistema de pensiones jubilar más universal y solidario, que aporte a mejorar las condiciones de vida de los adultos mayores y disminuya la desigualdad.

\section{ENVEJECIMIENTO Y SEGURIDAD SOCIAL EN AMÉRICA LATINA Y ECUADOR}

La población de América Latina, como la de Ecuador, ha empezado un proceso de envejecimiento que va acelerándose en el tiempo, y si bien ese proceso es diferente en cada país, la realidad es que todos los países experimentan cada año la mayor presencia de población en edad avanzada (ver Tabla 1). La política pública y la sociedad en su conjunto deben prepararse con celeridad para proteger a este grupo poblacional.

Ecuador cuenta todavía con una población mayoritariamente joven, si consideramos la proyección de la población menor o igual a 29 años para el 2015 (INEC, 2015). Este país, como el resto de países andinos y otros países de la región, sobre todo aquellos de menor desarrollo 
relativo, tiene un índice de envejecimiento menor al promedio regional. Según las proyecciones del Observatorio Demográfico para el año 2015 (CEPAL, 2014), Ecuador tendría 33,3 adultos mayores (de 65 años y más) por cada 100 personas menores de 15 años, mientras el promedio regional habría sido de 42,8 adultos mayores para ese mismo año. Ecuador forma parte, por tanto, de un grupo de países que todavía tienen abierta una ventana de oportunidad para establecer sistemas de protección social más adecuados para la vejez.

La seguridad social es una de las políticas más importantes para proteger a la población en edad avanzada, una vez que las personas se retiran del mercado laboral. Las prestaciones de salud y la pensión jubilar son particularmente importantes. La afiliación a la seguridad social en la región, sin embargo, es limitada, y se encuentran excluida sobre todo la población más pobre. Según las estimaciones de CEPAL (2013), para el 2011, el 45\% de la población asalariada de 15 años y más no estaba afiliada a la seguridad social y el $34 \%$ de esa población no estaba afiliada a un sistema de salud, siendo la población pobre la más afectada (ver Figura 1).

Ecuador no está al margen de esta problemática, y si bien, la población afiliada crece más de lo que crece el promedio de América Latina entre el 2002 y 2011, incluyendo la cobertura de los asalariados en los quintiles de ingreso más bajo, es este grupo de población el que menos cobertura relativa tiene (ver Tabla 2).

Entre los problemas que limitan el acceso a la seguridad social y por tanto al sistema de pensiones, tanto en la región como en Ecuador, podemos considerar: i) La persistencia de un mercado laboral heterogéneo con un abultado empleo informal no afiliado a la seguridad social, trabajadores campesinos y trabajadores por cuenta propia que por sus ingresos limitados y/o volátiles no han podido ser incorporados al sistema. En la región, para el 2011, un 47,7\% de trabajadores no agrícolas tuvieron empleo informal, y si bien la situación ha mejorado, sigue siendo una proporción importante de trabajadores no protegidos (OIT, 2012). Para Ecuador, el empleo en el sector informal para diciembre del 2015 llegó al 40,36\% (INEC, 2015b). ii) Muy relacionado con el problema anterior, está la alta desigualdad en la distribución del ingreso y la persistencia de la pobreza. La pobreza por ingresos en la región para el 2014 afectó al 28\% de la población según CEPAL, y el índice de Gini por ingresos para ese mismo año fue de o,486, el más alto respecto a otras regiones del mundo (2014c). Ecuador, para el 2014 registró un nivel de pobreza y desigualdad algo menor al promedio regional: $22.49 \%$ de incidencia de pobreza por ingresos, y un Gini por ingresos de 0,466 (INEC, 2015C). Las brechas de productividad laboral están por detrás de la desigualdad de ingresos. iii) La existencia de una gran proporción de mujeres a cargo de los servicios de cuidado en el hogar que no se encuentran afiliadas. Un tercio de las mujeres de 15 años y más que no estudiaba en la región, no generó ingresos propios en el 2011 (CEPAL, 2014c). iv) Limitaciones para el acceso de los jóvenes, que se encuentran sobrerrepresentados en el sector informal (Robles, 2012). v) Debilidades institucionales en las tareas de recaudación y control.

Aparte de estos problemas que explican sobre todo las dificultades de acceso a la seguridad social, existen los temas de solidaridad y sostenibilidad del financiamiento que son muy heterogéneos según el país, pero que merecen una profunda reflexión a la hora de pretender avanzar hacia un sistema más universal y equitativo, en momentos de transición demográfica. Esta situación marca una distancia muy grande con los sistemas de seguridad social europeos 
Tabla 2. Afiliación a sistemas de pensiones/salud entre asalariados de 15 años y más, según quintil de ingreso per cápita (\%)

\begin{tabular}{lcccc}
\hline & \multicolumn{2}{c}{ ECUADOR ZONAS URBANAS } & \multicolumn{2}{c}{ AMÉRICA LATINA } \\
\cline { 2 - 5 } QUINTILES & PENSIONES & SALUD & PENSIONES & SALUD \\
\hline 2002 & & & & \\
\hline Total & 42,0 & 45,0 & 46,1 & 54,4 \\
Quintil I & 17,6 & 18,1 & 17,3 & 27,8 \\
Quintil II & 24,9 & 26,9 & 28,3 & 37,3 \\
Quintil III & 34,3 & 36,3 & 39,2 & 47,5 \\
Quintil IV & 45,3 & 48,3 & 50,3 & 57,6 \\
Quintil V & 64,9 & 70,3 & 65,1 & 71,4 \\
& & & & \\
\hline 2011 & & & & 66,4 \\
Total & 64,7 & 66,2 & 55,4 & 44,0 \\
Quintil I & 30,1 & 33,2 & 24,4 & 53,2 \\
Quintil II & 44,6 & 47,7 & 37,9 & 61,1 \\
Quintil III & 58,1 & 59,7 & 49,0 & 69,5 \\
Quintil IV & 70,3 & 71,6 & 59,8 & 70,6 \\
Quintil V & 86,3 & 86,7 & 73,0 & \\
\hline
\end{tabular}

Fuente: CEPAL (2014a).

que cuentan con una mayor proporción de trabajadores en el sector formal, una mejor distribución del ingreso y un alto cumplimiento de las leyes de seguridad social, a pesar de que enfrentan un nivel relativamente mayor de envejecimiento poblacional $y$, actualmente, una coyuntura de crisis económica y desempleo todavía no resuelta. América Latina, sin embargo, cuenta todavía con una ventana de oportunidad en la mayoría de países, como en el caso de Ecuador, que todavía tiene una población relativamente joven, pero debe enfrentar los problemas estructurales de su desarrollo y mercado laboral. Es debido a esta compleja problemática que la región no ha logrado universalizar la seguridad social contributiva y ha ido introduciendo programas de protección social no contributiva - como las transferencias monetariaspara compensar esos déficits, conforme se verá más adelante.

A continuación se hace un breve recuento del desempeño social y la coyuntura económica de la región y Ecuador, para contextualizar los desafíos de las políticas de protección y seguridad social.

\section{DESEMPEÑO SOCIAL Y CONTEXTO ECONÓMICO EN AMÉRICA LATINA: AVANCES Y CUELLOS DE BOTELLA}

América Latina experimenta un período de desaceleración luego de un período de bonanza en lo que va del presente siglo. La Comisión Económica para América Latina, CEPAL (2015), estima que la región habría decrecido -0,4\% en el año 2015, y que prácticamente se estancará 
en el 2016, con un 0,2\% de crecimiento del Producto Interno Bruto, PIB, regional. Éste también es el caso de Ecuador, pues, aunque su tasa de crecimiento de la economía está sobre el promedio de la región, esta habría llegado apenas a 0,4\%, en el 2015, según la última previsión del Banco Central del Ecuador. Si bien han existido avances importantes, tanto en términos políticos por una mayor estabilidad de sus democracias, como en términos del desempeño social, quedan muchos desafíos pendientes en grupos poblacionales sensibles y altamente vulnerables como la población adulta mayor, que debe enfrentar un contexto de desaceleración económica. Esta sección presenta un breve balance del desempeño social y los principales cuellos de botella, particularizando la información sobre adultos mayores, para fundamentar la construcción de una agenda urgente para un sistema de pensiones en Ecuador, que puede ser extensivo a países de parecidas características socioeconómicas en la región.

En términos generales, la región ha experimentado un mejoramiento de sus indicadores sociales. La pobreza ha bajado persistentemente en lo que va del presente milenio, como se puede observar en la figura 2, si bien en los últimos años tiende a estacionarse. Ecuador tiene un desempeño similar, incluso con mayores avances en la reducción de pobreza y la desigualdad que el promedio regional (CEPAL, 2014a). Estos avances se explican no solo por el buen desempeño económico que la región experimentó en los años previos, dado por una coyuntura favorable en el precio de sus materias primas, sino por la creciente importancia macroeconómica y presupuestal del gasto y la política social. De hecho, Ecuador subió 5 puntos su participación del gasto social en el PIB entre 2006 y 2014, pasando de 4,2\% al 9,2\% (MCDS, 2014), sin considerar los subsidios a pensiones jubilares de la seguridad social (1,3\% del PIB aproximadamente para el 2012, según Naranjo (2013)) y el gasto en educación superior, mientras la región, partiendo de una participación promedio del gasto social más alta, subió 2 puntos en el pib, del 13,2\% al 15\% entre 2006 y 2013 (CEPAL, 2014c), (ver Figura 2).

Si bien se ha hecho un esfuerzo importante en incrementar el gasto social de la región, existe una recaudación tributaria relativamente baja respecto a los países de la oCDE, y la que existe es regresiva (CEPAL, 2014C). Ello limita la capacidad redistributiva del Estado y la posibilidad de corregir ex post la dinámica excluyente de los mercados, particularmente del mercado laboral. Cabe aclarar que Ecuador avanzó mucho en recaudación tributaria aumentando 5 puntos entre el 2006 (9,1\% del PIB) y el 2014 (14,3\% del PIB), según la información estadística del Banco Central; sin embargo, sigue lejos de la recaudación tributaria promedio observada en los países de la región que para el 2014 alcanzó el 17,9\% del PIB (CEPAL, 2015). Las necesidades para financiar los gastos que implicaría impulsar políticas relevantes como la universalización de la seguridad social, de la educación y de los servicios de salud, siguen siendo muy grandes. Por tanto, los análisis y las decisiones sobre prioridades, vías de transición hacia esas metas, fuentes de financiamiento y mecanismos de progresividad son claves para lograr los resultados anhelados. Más aún cuando se enfrenta un ciclo económico desfavorable.

Los avances sociales de la región también alcanzaron al mercado laboral, sobre todo en lo referente a la tasa de desempleo que ha venido disminuyendo; sin embargo, el empleo informal, muy correlacionado con pobreza y baja productividad, sigue siendo un gran problema estructural. Como se observó en la sección previa, según la OIT (2012) para el 2011 el empleo informal no agrícola afectaba al 47,7\% de la Población Económicamente Activa (PEA) de la región, 
Figura 2. Evolución de la pobreza y la indigencia 1990-2014 (en porcentaje de personas) en América Latina

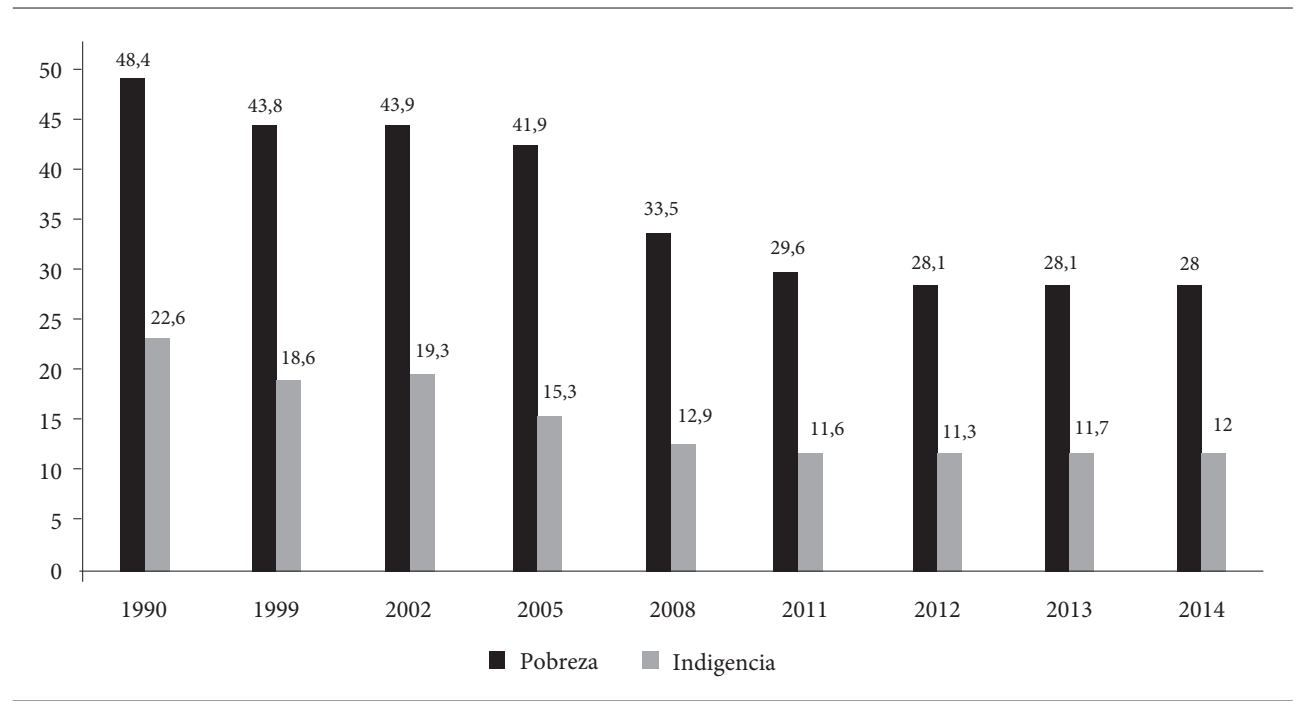

Fuente: CEPAL (2014).

pese a las mejoras de los últimos años (OIT, 2012; Banco Mundial, 2012), y en Ecuador el empleo en el sector informal llegó al 40,36\% del total de empleados, lo que explica, en parte, la limitada movilidad social a través del trabajo y la limitada afiliación a la seguridad social. Pese a los avances evidenciados en el desempeño social, América Latina sigue siendo la región más desigual del mundo (CEPAL, 2014a), y la pobreza por ingresos para el 2014 siguió afectando a un poco más de uno de cada cuatro personas (INEC, 2014).

En este escenario de avances sociales, pero limitada dinámica económica, mayor envejecimiento de la población, pero con un punto de partida de mayor población joven, es importante desarrollar políticas para enfrentar las rigideces estructurales y las contingencias en un mundo de mayor incertidumbre para las etapas de la vida de mayor vulnerabilidad. El sistema de seguridad social, y particularmente el sistema de pensiones merece particular consideración entre las políticas públicas para personas de edad avanzada a fin de contrarrestar su empobrecimiento en una etapa de la vida de mayor necesidad, sobre todo para la atención de salud y cuidado.

En la siguiente sección se analizan las políticas de protección social contributiva y no contributiva, particularmente los sistemas de seguridad social y las transferencias monetarias.

\section{POLÍTICAS DE PROTECCIÓN SOCIAL}

Las políticas de protección social son una dimensión muy importante de las políticas sociales destinadas a proteger a la población contra los distintos riesgos y contingencias que se presentan durante su ciclo de vida. Estas políticas, sin embargo, son solo un eje de varias 
dimensiones de política social que deben caminar de la mano, como las políticas de promoción social, incluidas aquellas que fortalecen capacidades como educación y salud; pero que también favorecen el acceso a activos como vivienda, tierra, agua, financiamiento, políticas laborales y de empleo, entre otras (Robles, 2012). Las políticas sociales, por otra parte, no pueden ser políticas netamente sectoriales, deben ser políticas altamente articuladas a las políticas económicas fundamentales como la política fiscal, la política productiva, la política macroeconómica, sobre todo cuando se trata de enfrentar problemas estructurales como la desigualdad y el empleo informal.

Si bien se han hecho esfuerzos muy importantes, sobre todo en los últimos años, tanto en Ecuador como en otros países de la región, no se han podido garantizar derechos universales de ciertos servicios sociales básicos como salud, seguridad social y educación (excepto educación primaria). El país, como la mayoría de países de la región, cuenta con un Estado de bienestar en ciernes y, por tanto, tiene desafíos muy grandes para enfrentar los problemas de desigualdad. Esta realidad, sumada a la heterogeneidad del mercado laboral, con un sector informal abultado y muy relacionado con pobreza y baja productividad, hace que los factores de movilización social clásicos no operen de igual manera en las sociedades latinoamericanas. La política de protección social es una necesidad para todos y particularmente para la población excluida que tiene muy pocas posibilidades alternativas de protección en el sector privado, o en las redes familiares y comunitarias para enfrentar contingencias, menos aún durante una etapa del ciclo de vida sensible como la vejez.

Los debates sobre sistemas de protección social actual en la región, no están restringidos a las crisis o a la pobreza, sino que además confrontan derechos básicos universales e incluyen una complejidad de políticas y coordinaciones interinstitucionales, aunque ciertamente con énfasis diferenciados según la historia de cada país en la consolidación de las políticas sociales, y sus características socioeconómicas y demográficas.

Si bien el concepto «protección social» tiene distintas interpretaciones (Robles, 2012; Naranjo, 2013) y existen varias experiencias en la región, este trabajo considera su sentido más universal en el aseguramiento de derechos básicos y la protección frente a contingencias (Ceccini y Martínez, 2011; Robles, 2012). Supone, además, en sociedades altamente desiguales como las latinoamericanas, un financiamiento solidario que apoye a la igualdad y disminuya la pobreza. Acogiendo la propuesta de Ceccini y Martínez (2011), los componentes básicos de este sistema son: la protección social contributiva, que en este trabajo se asociará con la seguridad social contributiva; la protección social no contributiva, que en este documento considerará las transferencias monetarias no contributivas; y la regulación del mercado laboral destinada a proteger a los trabajadores contra riesgos y contingencias. Si bien existen otros estudios como el de Naranjo (2013), que consideran como políticas de protección y promoción social no solo las políticas aquí consideradas, sino también las políticas sociales sectoriales y focalizadas como educación, salud, vivienda y desarrollo humano, inclusión económica social y empleo, en este artículo se considera a la protección social en el sentido de Ceccini y Martínez (2011), siendo solo una parte de la política social que es mucho más amplia e integral, e incluye no solo la política sectorial en educación, salud, vivienda, sino políticas transversales como la inclusión y promoción económica y social, el empleo y su necesaria articulación a la política económica. 
En este marco, este trabajo se concentra en el sistema de protección social contributivo y no contributivo, y particularmente en el sistema de pensiones de la población en edad avanzada. Con tal fin, los siguientes apartados analizan el sistema de seguridad social, particularmente su sistema de pensiones jubilares y las transferencias monetarias para adultos mayores en el nivel regional, y colocan el caso ecuatoriano como una experiencia relevante para países de desarrollo relativo medio en la región.

\section{LA SEGURIDAD SOCIAL}

Un sistema de seguridad social se basa normalmente en un sistema contributivo y, excepcionalmente, no contributivo, financiado sobre todo por las contribuciones de los trabajadores formales, en el que también aportan los empleadores y el Estado. Generalmente, su protección contempla una cartera de prestaciones destinadas a cubrir las contingencias de desempleo, maternidad, enfermedad, discapacidad, viudez, vejez y muerte. Este sistema es clave no solo para proteger a los trabajadores y sus familias del riesgo de contingencias sino también para apoyar la equidad. En América Latina existe una heterogeneidad de modelos; según Mesa-Lago (2004), existen al menos tres grupos de países en consideración del origen y desarrollo de sus sistemas de seguridad social: i) los países pioneros — primer cuarto del siglo $\mathrm{xx}$ - de mayor desarrollo relativo, mercados laborales más formales y mayor cobertura no solo de la seguridad social, sino de educación y salud, como Uruguay, Argentina, Chile, Brasil, Cuba y Costa Rica; ii) los países intermedios que desarrollaron sus sistemas alrededor de la mitad del siglo $\mathrm{xx}$, con mayor proporción de trabajadores en el sector informal y menor expansión de otros servicios como educación y salud, donde se sitúan los países andinos, entre ellos Ecuador, además de México y Panamá; y iii) los países tardíos, que impulsaron sus sistemas entre los años sesenta y setenta del siglo xx y que tienen menor desarrollo social relativo, alta participación de los trabajadores en el sector informal y alta pobreza, donde se encuentran sobre todo los países centroamericanos, caribeños y Paraguay.

Varias reformas han ocurrido en el tiempo, particularmente entre los años setenta y noventa (Mesa-Lago, 2004). Algunos países como Chile, Bolivia, México, El Salvador, República Dominicana y Nicaragua, privatizaron servicios sociales y promocionaron la capitalización individual. Otros países impulsaron reformas tanto en los seguros públicos como privados, como en los casos de Perú y Colombia. Otros emprendieron reformas mixtas como en el caso de Argentina, Uruguay, Costa Rica. También se dieron reformas paramétricas como en Brasil, Cuba, Guatemala, Haití. En Ecuador se mantuvo el sistema de seguridad social público, pese a los varios intentos de privatizarlo.

Actualmente existe una gama muy variada de modelos de seguridad social que fluctúa entre público, privado y mixto. En lo que a cotizaciones de pensiones y jubilaciones se refiere, tenemos: i) sistema de reparto (beneficio definido); ii) capitalización parcial colectiva; y iii) capitalización plena (contribución definida), (Mesa-Lago, 2004). Ecuador tiene un sistema híbrido de reparto y capitalización parcial colectiva. 


\section{LAS TRANSFERENCIAS MONETARIAS}

Las transferencias monetarias en América Latina, como parte del sistema no contributivo de protección social, se financian del presupuesto fiscal; es decir, del ingreso tributario general. Sus orígenes se encuentran en la compensación a los más pobres frente a los ajustes fiscales implantados luego de la crisis de la deuda de los años ochenta, pero su visión ha cambiado en el tiempo y hoy conviven varios enfoques que abarcan, desde los enfoques más comprensivos para enfrentar la multidimensionalidad de los factores que influyen en la pobreza, hasta aquellos que apuntan a cubrir derechos universales y plantean sendas articulaciones con la política social y económica.

Estos programas han tenido una amplia difusión en América Latina y en la actualidad al menos 18 países los incluyen en sus políticas sociales (Robles, 2012), debido posiblemente a su bajo costo, amplia cobertura, acceso inmediato a ingresos por parte de los hogares en situación de pobreza (Robles, 2012; Ceccini y Martínez, 2011), y procesos de difusión de actores internacionales (Osorio, 2014), entre otros. Existen diferentes grupos de programas, según sus objetivos claramente diferenciados (Robles, 2012): aquellos que buscan asegurar un ingreso básico para la población en situación de vulnerabilidad (crisis o riesgos) articulado en la mayoría de casos a la promoción de la acumulación de capital humano (educación y salud) de los hijos en los hogares pobres; aquellos que buscan erradicar la pobreza atendiendo a su naturaleza multidimensional, coordinándolos con políticas sociales y económicas; y aquellos que buscan promover derechos sociales y económicos ciudadanos de carácter universal al margen de su situación de pobreza, como son los que buscan promover la renta básica, o los que buscan universalizar el sistema de seguridad social y particularmente el sistema de pensiones, articulando instrumentos no contributivos.

Este documento se centra en el último objetivo de las transferencias monetarias, como mecanismo de apoyo a la transición hacia un sistema más integrado de seguridad social, particularmente dirigido a proteger a la población adulta mayor como una de sus misiones fundamentales. En la región, las transferencias monetarias, materializadas como pensiones no contributivas para quienes no tienen prestaciones contributivas han sido ampliamente utilizadas en los casos de Argentina, Brasil, Chile, Colombia, Costa Rica, El Salvador, Paraguay, Uruguay (Robles, 2012), Bolivia y Ecuador. La participación de este tipo de transferencias ha crecido en el tiempo y son parte importante de las pensiones en la población adulta mayor (ver Tablas 3 y 4 ).

En consideración de su gran difusión, sus demandas de financiamiento y las demandas de otras políticas, es importante desarrollar una articulación más adecuada con el sistema de seguridad social contributivo, mirar los mecanismos de progresividad en función de los tipos de financiamiento y plantear un estrategia de transición que aliente el trabajo digno y la seguridad social contributiva, para que dinámicamente hagan menos falta estos mecanismos no contributivos. A continuación se expone brevemente el caso ecuatoriano, para provocar una reflexión sobre la protección a adultos mayores, particularmente a través de un sistema de pensiones, en países de desarrollo relativo intermedio en la región con alta participación del sector informal. 
Tabla 3. Cobertura de los programas de transferencias condicionadas en América Latina y el Caribe

\begin{tabular}{lc}
\hline AÑo & $\begin{array}{c}\text { COBERTURA DE LOS PROGRAMAS DE TRANSFERENCIAS CONDICIO- } \\
\text { NADAS (EN PORCENTAJES DEL TOTAL DE LA POBLACIÓN) }\end{array}$ \\
\hline 2000 & 5,7 \\
2005 & 13,5 \\
2008 & 15,4 \\
2009 & 18,2 \\
2010 & 18,7 \\
2011 & 19,5 \\
2012 & 20,3 \\
\hline
\end{tabular}

Fuente: CEPAL (2014).

Tabla 4. Cobertura de los programas de pensiones en América Latina y el Caribe

\begin{tabular}{lc}
\hline AÑo & $\begin{array}{c}\text { COBERTURA DE LOS PROGRAMAS DE PENSIONES (EN POR- } \\
\text { CENTAJES DEL TOTAL DE LA POBLACión) }\end{array}$ \\
\hline 2008 & 33,2 \\
2009 & 35,8 \\
2010 & 37,2 \\
2011 & 38,3 \\
2012 & 40,7 \\
\hline
\end{tabular}

Fuente: CEPAL (2014).

\section{CASO ECUATORIANO}

Ecuador, como se anotó previamente, es un país con una población relativamente joven en América Latina, pero con un proceso dinámico de envejecimiento. De acuerdo a las proyecciones de población del INEC (2015), el 54,1\% de la población es menor o igual a 29 años, y como advirtió anteriormente su tasa de envejecimiento $(33,3)$ proyectada al 2015 es alrededor de 9 puntos más baja que la tasa de envejecimiento promedio de la región $(42,8)$, según el Observatorio Demográfico de CEPAL (2014). Según las proyecciones poblacionales de la CELADE (2015), el país contará con una ventana de oportunidad por un par de décadas más, mientras siga aumentando la población en edad productiva - población entre 15 a 64 años, para el año 2015 fue el 62,5\% de la población total- y baje la tasa de dependencia de la población - población menor de 15 años y población mayor o igual a 65 años respecto a población de 15 a 64 años-. Pero pese a contar con una población relativamente joven, la población adulta mayor, de 65 años y más, está creciendo y crecerá más aceleradamente que el conjunto de la población (ver Figura 3). Por ello, es fundamental que el Estado, las familias y las comunidades estén preparados.

De este modo, frente al proceso de envejecimiento inexorable que va ocurriendo en la población ecuatoriana, es importante aprovechar la ventana de oportunidad demográfica para hacer reformas a la seguridad social y a la política social dirigidas a adultos mayores que nos 
lleven a un nivel de protección social más adecuado, con mayor equidad y sostenibilidad, así como políticas económicas, laborales y productivas, que confronten los problemas estructurales del mercado laboral advertidos. El sistema de pensiones jubilares tanto contributivas como no contributivas es un eje muy importante de la política de protección social para promover un envejecimiento más digno de la población. En términos de desarrollo social, si bien se han dado avances en Ecuador, al igual que en América Latina, como se refirió previamente, los adultos mayores todavía enfrentan situaciones de pobreza y desprotección social complejos. Según el censo de población del año 2010, el 45\% de la población adulta mayor se encontraba en condiciones de pobreza y extrema pobreza por necesidades básicas insatisfechas. Sólo uno de cada cuatro adultos mayores tenía pensión jubilar contributiva (26\%), el 22\% no tenía ningún seguro, y el 52\% recibía una pensión asistencial no contributiva en el 2014 (MCDS, 2014b). Quedando, por tanto, un reto muy grande para avanzar hacia la universalización de la pensión jubilar con una estrategia de financiamiento sostenible y solidaria.

Desde la perspectiva de las políticas sociales, Ecuador ha vivido una importante dinámica que ha seguido el paradigma del Estado de bienestar que busca garantizar la universalización de servicios clave como educación, salud y seguridad social, en el contexto de un pacto social expresado en la Constitución de 2008, que se plantea la búsqueda de una sociedad más justa y el objetivo del Buen Vivir para su población. En términos prácticos, esto se ha expresado en una mayor atención al sector social, y el impulso a políticas redistributivas que contaron con mayores ingresos fiscales. De hecho, el presupuesto social más que duplicó su participación en el PIB, del 4,2\% en el 2006 al 9,2\% en el 2014. Las políticas de educación, de salud, de vivienda, de inclusión social y económica, así como las políticas de protección social y de seguridad social, dinamizaron propuestas y ampliaron coberturas (Naranjo, 2013, Sánchez, 2012).

Los avances en el desempeño y política social, sin embargo, enfrentan una coyuntura económica adversa en el país, como en el resto de la región, debido al abaratamiento del precio del petróleo, su principal producto de exportación, y por la grave apreciación monetaria que ha reducido la competitividad de la producción ecuatoriana. Las previsiones de crecimiento económico, según el Banco Central del Ecuador, para el 2015, son de apenas el o,4\%. El 2016 tampoco aparece muy alentador en términos de crecimiento, lo cual pone en tensión los logros avanzados en el campo social, planteando un gran desafío para el financiamiento sostenible de la política social y, particularmente, de las pensiones jubilares en el mediano y largo plazo. En este contexto, el sistema de pensiones jubilares merece particular consideración en el marco de políticas sociales y particularmente de protección social dirigidas a la población en edad avanzada, que prevengan su empobrecimiento y permitan un envejecimiento con dignidad.

A continuación se considerará la situación de la seguridad social, particularmente de las pensiones jubilares contributivas, y de las transferencias monetarias, concentradas en el tema de pensiones jubilares no contributivas.

\section{LA SEGURIDAD SOCIAL EN ECUADOR Y LOS ADULTOS MAYORES}

El tipo de Seguridad Social en el caso ecuatoriano corresponde a las características del grupo intermedio de países en la tipología de Mesa-Lago (2004); es decir, un país con una proporción 
Figura 3. Tasa de crecimiento de la población mayor o igual a 65 años y de la población total en Ecuador

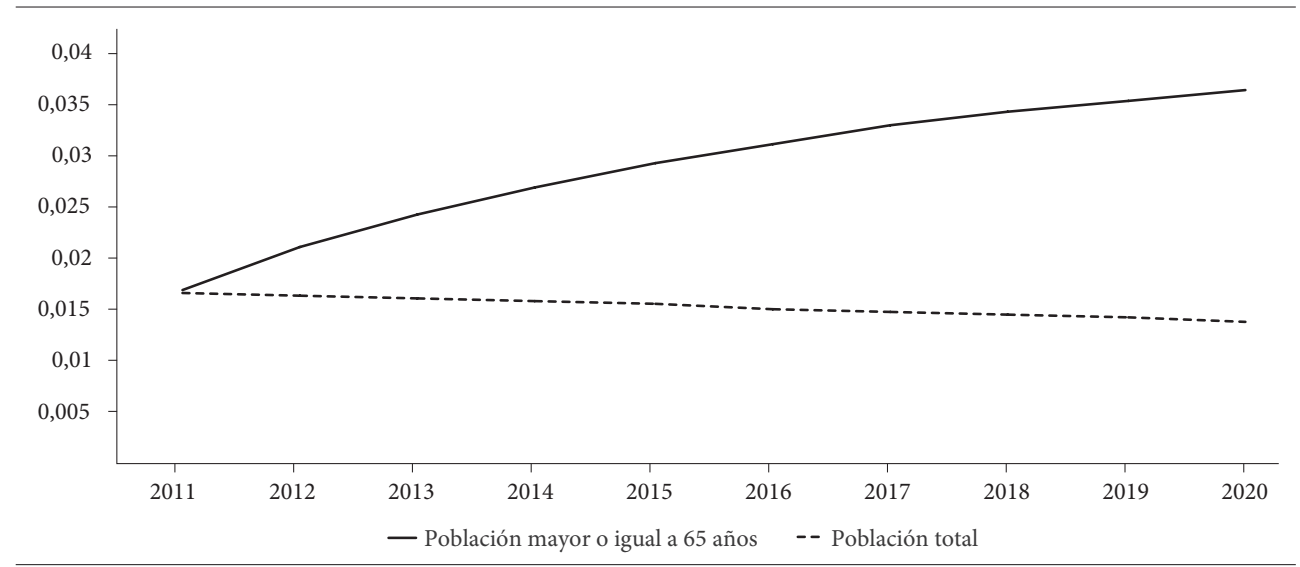

Fuente: INEC (2015a).

Figura 4. Participación de la PEA afiliada a la Seguridad Social en Ecuador (\%)

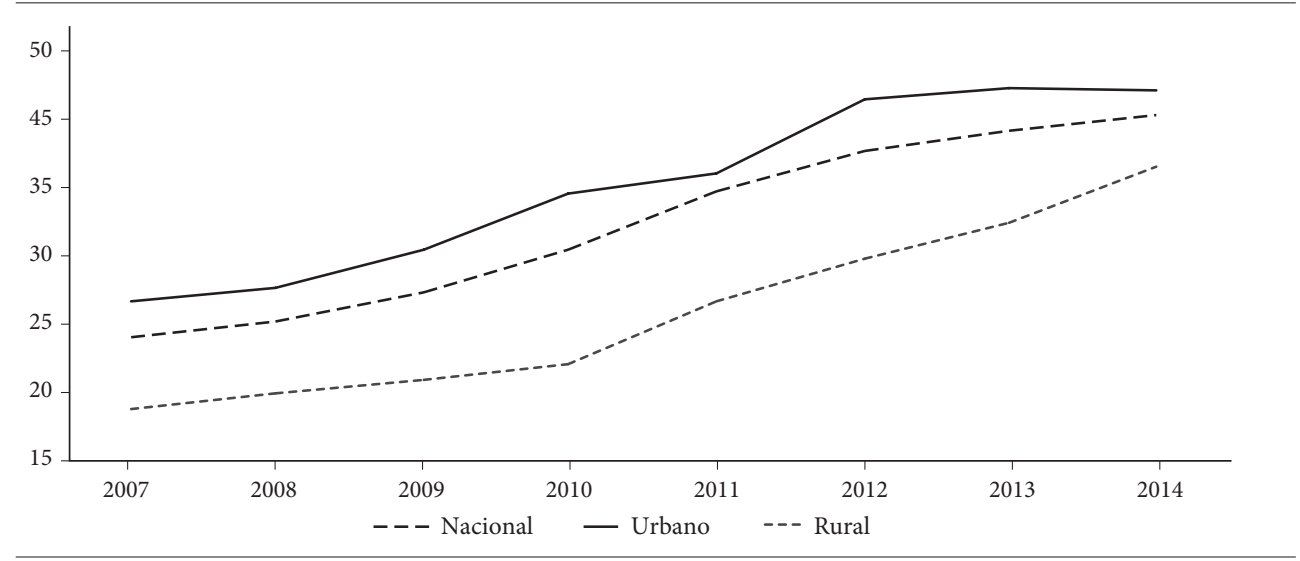

Fuente: INEC (datos a diciembre de cada año). Ministerio Coordinador de Desarrollo Social de Ecuador.

importante de trabajadores informales (40,36\% de la PEA en diciembre del 2015) y por consiguiente con limitaciones en la cobertura de su sistema de seguridad social. La PEA afiliada a la seguridad social no llega a la mitad (44,34\% para el 2014) pese a los esfuerzos realizados por ampliar la cobertura en los últimos años, tanto en el sector urbano como rural (ver Figura 4). El gran crecimiento que de todas maneras ocurrió en la cobertura de la población afiliada a la seguridad social en la última década, obedece a políticas activas conducentes a captar mayor población afiliada. Entre estas políticas se puede mencionar: la penalización de la no afiliación a la seguridad social para los patronos incumplidos, que fue incorporada en virtud de una consulta popular realizada en el 2011; el incremento de los beneficios de la afiliación, que, 
entre otros, extendió la cobertura de servicios de salud a hijos menores de 18 años y cónyuges; la reducción del período requerido para acceder a las prestaciones de salud; el apoyo en prestadores privados de salud; la reactivación de los créditos quirografarios e hipotecarios, que son parte de los beneficios de la seguridad social en el caso ecuatoriano; por último, la mayor activación de las inspectorías del trabajo y campañas puerta para asegurar el cumplimiento de las normas de afiliación.

Según la Constitución de 2008, el sistema de seguridad social ecuatoriano, como en la mayoría de sistemas de seguridad social de la región, debe atender las contingencias de enfermedad, maternidad, paternidad, riesgos del trabajo, cesantía, desempleo, vejez, invalidez, discapacidad, muerte y aquellas que defina la ley. Existe un pacto tácito de que el sistema de seguridad social en Ecuador es público y universal, conforme lo define la Constitución (2008) que fue aprobada por la sociedad ecuatoriana a través de un referéndum en el 2008. Este pacto no es reciente, se ha ido forjando en el tiempo, si bien la población beneficiaria ha sido acotada, hay la aceptación mayormente generalizada entre los principales actores de la sociedad de que el sistema de la seguridad social debe ser público y debe cubrir a todos, de manera progresiva. En este sentido, se podría decir que hay un pacto cercano al tipo de pacto definido por el enfoque neoestructuralista histórico de políticas, conforme lo refieren Maldonado y Palma (2013), cuando existe un acuerdo en torno a una política pública basado en paradigmas compartidos por los principales actores. En ese contexto, aquí se tratará particularmente el tema de pensiones.

El sistema de seguridad social en Ecuador se basa en un sistema contributivo y, excepcionalmente, no contributivo, financiado por las contribuciones de los trabajadores, los empleadores y el Estado. Es un sistema híbrido de reparto y capitalización colectiva parcial. La seguridad social es provista por tres instituciones públicas y autónomas como son el Instituto Ecuatoriano de Seguridad Social, IESs, el Instituto de Seguridad Social de las Fuerzas Armadas, ISSFA, y el Instituto de Seguridad Social de la Policía, ISSPOL. Si bien existen prestadores privados de seguros de salud, de pensiones, entre otros, los asegurados se encuentran sobre todo en la seguridad social pública. La seguridad social contempla una cartera de prestaciones destinadas a cubrir las contingencias de desempleo, maternidad, enfermedad, discapacidad, viudez, vejez y muerte. El IEss se articula mediante un seguro general para la población civil, que afilia obligatoriamente a los trabajadores dependientes y voluntariamente a quien realiza otro tipo de actividades e incluye un régimen especial para el trabajo en el campo y la pesca artesanal, a cargo del Seguro Social Campesino. El IEss maneja los siguientes seguros: el seguro de pensiones (invalidez, vejez y muerte), el seguro campesino, el seguro de salud, el seguro de riesgos del trabajo y el seguro de cesantía.

Uno de los problemas del sistema de seguridad social en Ecuador es su limitada cobertura, que como se anotó, si bien ha mejorado visiblemente en los últimos años, todavía mantiene un déficit importante. Los problemas de la cobertura de la afiliación de la seguridad social tienen que ver como lo interpreta Mesa Lago (2014), con la estructura del mercado laboral ecuatoriano y la presencia de una alta informalidad, y relacionado con ello, con la situación de pobreza y desigualdad, que pese a las mejoras sociales - bajaron 15 y 7 puntos respectivamente entre 2006 y 2014 - todavía persisten en el país. Si bien existen problemas de evasión y existen grupos profesionales independientes que no están afiliados, el déficit de cobertura afecta 
sobre todo a los trabajadores del sector informal, que es donde se encuentran la población más pobre y los trabajadores por cuenta propia. El empleo en el sector informal —entiéndase éste al empleo ocurrido en empresas que no cuentan con registro único de contribuyente- en la última década ha afectado a alrededor de cuatro de cada diez personas ocupadas (INEC, 2015). El sector informal está altamente relacionado con las condiciones de pobreza y baja productividad (Banco Mundial, 2012).

Esta realidad plantea un desafío estructural importante, pues ya no se trata solamente de un sector económico que no quiere cumplir con las normas, sino de un sector que por condiciones estructurales tiene bajos niveles de productividad y, por tanto, bajos niveles de ingresos para poder cumplir con los costos de afiliación y formalización en general. La incorporación de este sector requiere de políticas dirigidas a mejorar sus niveles de productividad, la consideración de un tratamiento especial en los parámetros de contribución y alcance de beneficios, de políticas de apoyo al financiamiento o subsidio a la contribución para los sectores de menores ingresos, al menos en un período inicial de transición, y no solo de normas y exigencia de cumplimiento. Adicionalmente, otros grupos de baja cobertura de afiliación son las mujeres a cargo de las tareas del hogar sin remuneración, cuyo volumen equivale a algo más de la mitad de la población actualmente afiliada (MCDS, 2014) y el grupo de los jóvenes.

En el caso de las mujeres, la reciente Ley Orgánica para la Justicia Laboral y Reconocimiento del Trabajo en el Hogar (2015), abrió la opción de la afiliación al IEss de las personas que realicen trabajo no remunerado en el hogar para que sean protegidas de las contingencias de vejez, muerte e invalidez, con la contribución de la unidad económica familiar y un subsidio progresivo del Estado a la cotización hasta cuando los ingresos iguales o sean inferiores al 150\% del salario básico unificado (Resolución CD N. ${ }^{\circ} 492,2015$ ). Quedan, sin embargo, pendientes, la reglamentación de la incorporación de los trabajadores independientes y el tratamiento y posible subsidio a la cotización de aquellos de menores ingresos. De este modo, existe un desafío muy importante para pensar en la universalización de la seguridad social y su financiamiento en Ecuador.

Las limitaciones históricas en la afiliación a la seguridad social han provocado una baja cobertura en las pensiones jubilares, que enfrenta una mayor dinámica de envejecimiento poblacional, como se analizó previamente. Apenas 1 de cada 4 adultos mayores, recibe pensión jubilar contributiva, 2 reciben pensión jubilar no contributiva y 1 no tiene ninguna pensión. Esta situación pone una presión muy importante en las necesidades de financiamiento, por lo que es fundamental encontrar las fuentes y las corresponsabilidades más adecuadas con criterios de equidad y solidaridad, para evitar el empobrecimiento de este grupo poblacional.

Más allá de la limitada cobertura, el sistema de la seguridad social ecuatoriano y su sistema de pensiones jubilares, históricamente ha mantenido problemas de regresividad (Durán, 2008), porque ha subsidiado más a la población afiliada que a la población no afiliada, normalmente la más pobre. Hasta el primer trimestre del 2015, el Estado ecuatoriano subsidió el $40 \%$ de las pensiones jubilares del IEss y hasta la presente fecha subsidia el 60\% de las pensiones jubilares del ISSFA y el ISSPOL. Este subsidio representó para el 2012, 1039,8 millones de dólares, lo que fue el 1,3\% del piB en ese año (Naranjo, 2013). Si bien en el contexto de la creación de un programa de transferencias monetarias (Bono de Desarrollo Humano) también se crearon pensiones no contributivas destinadas a los adultos mayores más pobres, sin afiliación 
a la seguridad social, en el 2012 estos recursos apenas representaron un quinto de las transferencias del Estado por pensiones a la seguridad social (MCDS, 2014C).

En abril del 2015, sin embargo, el gobierno retiró el subsidio del 40\% a las pensiones jubilares del IEss, reservando el subsidio a las pensiones jubilares sólo para cuando éste sea necesario, tanto para el Seguro Social Obligatorio como para el Seguro Social Campesino (Ley orgánica de Justicia Laboral y Reconocimiento del Trabajo en el Hogar, 2015). De este modo, queda pendiente pensar en la sostenibilidad futura del financiamiento de las pensiones jubilares, sabiendo que el Estado, según la Constitución ecuatoriana, debe garantizarlas. En el caso de los seguros de las Fuerzas Armadas y Policiales, se mantiene, sin embargo, el subsidio del $60 \%$ a las pensiones, considerando, adicionalmente, que los topes de pensiones jubilares en el ISSFA e ISSPOL son más del doble del tope de las pensiones en el caso del IESS, aspectos que ameritan mayor discusión. De este modo, resulta importante pensar en el financiamiento de largo plazo del sistema de seguridad social que todavía adolece de insuficiente cobertura, sobre todo para población que requerirá del subsidio del Estado para su cotización, hasta que mejoren las condiciones estructurales del mercado laboral y de la producción de una amplia población rural y urbana. Ello plantea el gran desafío de incluir a los jóvenes en empleos dignos, para que puedan contribuir a la seguridad social oportunamente y puedan ser protegidos durante su ciclo de vida.

En Ecuador, por otra parte, los servicios de salud se entregan a través de un sistema mixto. En el ámbito público, existe la atención a salud manejada por el Estado a través de una red de hospitales y centros de salud del Ministerio de Salud Pública financiado directamente por el presupuesto del Estado, y la atención a la salud brindada por el IESS, el ISSFA, el ISSPOL para su población afiliada y sus beneficiarios cubiertos (hijos hasta los 18 años y cónyuges), donde el Estado también está obligado a hacer transferencias para cubrir los gastos correspondientes a enfermedades catastróficas y la atención a los adultos mayores. A pesar de la insuficiencia del sistema de seguridad social ecuatoriano para cubrir las necesidades de pensiones jubilares contributivas, Ecuador, al igual que otros países de la región, ha avanzado en materia de protección social no contributiva, como se analiza en la siguiente sección.

\section{TRANSFERENCIAS MONETARIAS EN ECUADOR: EL CASO DE LOS ADULTOS MAYORES}

Las transferencias monetarias no contributivas enfocadas en los más pobres como respuesta a la crisis económica de fines de los años noventa y como compensación por la reducción de subsidios de los combustibles, aparecen en Ecuador en 1998. El programa cuenta con dos componentes: una transferencia para los hogares pobres, llamada actualmente Bono de Desarrollo Humano ( $\mathrm{BDH})$, que es entregada a las mujeres en representación del hogar y que se condiciona a la atención escolar y control de salud de los hijos; y una transferencia, denominada Pensión Asistencial para adultos mayores y personas con discapacidad.

El programa, en su parte orientada hacia los hogares pobres, fue incorporando un enfoque dirigido a enfrentar la pobreza de manera más integral. Incorporó a la condicionalidad del bdh, la posibilidad de un anticipo acumulado, llamado Crédito de Desarrollo Humano para 
Figura 5. Bono de Desarrollo Humano y Pensión Asistencial por tipo de beneficiario en Ecuador (en miles)

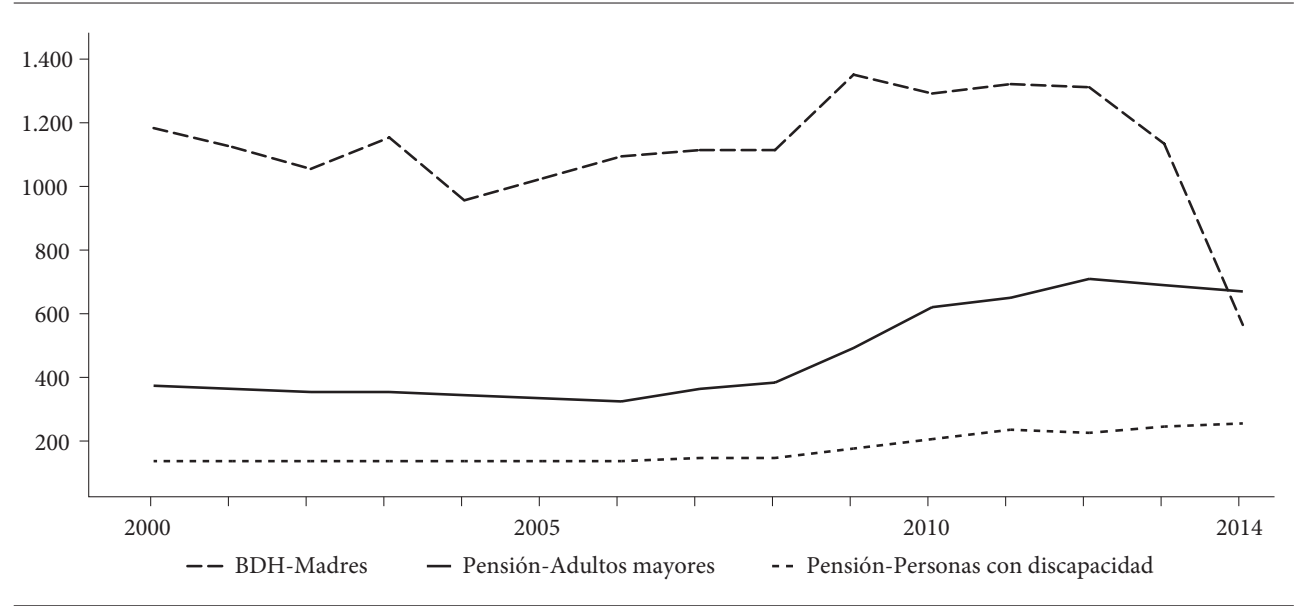

Fuente: MIES-RIES (2000-2014)

actividades productivas que, junto a programas de capacitación, becas escolares, acceso a vivienda, servicios de salud, fomento del empleo digno, desarrollo rural y fortalecimiento de la economía popular y solidaria, buscó apoyar una movilidad más integral (MCDS, 2009; MCDS, 2012; MCDS, MCDS, 2014; SENPLADES, 2014) (ver Figura 5).

En la última década el programa incrementó tanto la magnitud del Bono de Desarrollo Humano, BDH, como la cobertura, en parte por la actualización del registro social mantenido para identificar los hogares pobres como por la decisión política de ampliar la cobertura en los grupos en situación de mayor vulnerabilidad como adultos mayores y personas con discapacidad, como se observa en la figura 5. A partir del 2013 se tomó la decisión de focalizar el BDH en hogares de mayor pobreza y se bajó la línea de corte de los beneficiarios del BDH, por ello se observa un quiebre en la cobertura de madres beneficiarias del $\mathrm{BDH}$.

En el caso de las pensiones asistenciales para adultos mayores, se ha buscado conectarlas con el sistema de seguridad social, concibiéndolas como pensiones jubilares no contributivas, en una estrategia de progresividad que empieza con la atención a los más pobres. Esta pensión jubilar no contributiva cubrió al 52,2\% de la población adulta mayor en el 2014 (MCDS, 2015), compensando la gran subcobertura de la pensión jubilar contributiva, que apenas cubre a un cuarto de la población adulta mayor. Claramente, la pensión jubilar no contributiva para la población en edad avanzada y de mayor pobreza es importante en una transición hacia la universalidad, para proteger los ingresos de la población de mayor vulnerabilidad en la etapa final de su ciclo de vida. Sin embargo, debido al peso de su financiamiento, es fundamental activar políticas laborales, económicas y productivas más allá de las normas de afiliación y su cumplimiento. De este modo, debería ir disminuyendo en el tiempo la proporción de la pensión jubilar no contributiva.

Existe todavía un desafío importante en cuanto a la integración de un sistema de seguridad social contributivo que también incluya las pensiones jubilares no contributivas bajo 
un mecanismo de adecuada progresividad y sostenibilidad en el tiempo. Ecuador y los países de la región con sistemas de seguridad social con algunas características similares, tienen un desafío pendiente para lograr integrar sistemas de seguridad social más comprensivos hacia la universalidad, incluyendo la seguridad social contributiva y la protección social no contributiva, que consideren mecanismos de progresividad de los recursos públicos, solidaridad y sostenibilidad adecuados.

A continuación, se anotan algunas conclusiones y criterios que pueden apoyar ese proceso, pensando sobre todo en un sistema de pensiones más progresivo y sostenible, parte fundamental de un proyecto de protección social integral, universal y solidaria para la vejez.

\section{CONCLUSIONES Y CRITERIOS PARA LA CONSTRUCCIÓN DE UNA AGENDA}

La población de América Latina tanto como de Ecuador ha venido envejeciendo. Ecuador, sin embargo, pertenece a un grupo de países, al interior de la región, con población relativamente joven, por lo que tiene todavía una ventana de oportunidad de un par de décadas para preparar sus políticas sociales y, particularmente, su sistema de protección y seguridad social para apoyar un envejecimiento digno de su población.

Al igual que la región, Ecuador avanzó en el desempeño y la política social disminuyendo la pobreza y la desigualdad, sin embargo, subsisten desafíos muy grandes para lograr mayor protección social, inclusión y equidad, sobre todo en poblaciones en crecimiento como los adultos mayores, para quienes la sociedad, las familias y el Estado no están suficientemente preparados para atender. El sistema de protección y seguridad social es muy importante para proteger esa etapa sensible de la vida y evitar la pobreza y sus consecuencias en la calidad de vida, cuando más se necesita de cuidado y atención de salud. Sin embargo, todavía existe una gran proporción de adultos mayores desatendido en la región y Ecuador sin ninguna pensión jubilar. En Ecuador solo uno de cada cuatro adultos mayores recibe pensión jubilar contributiva, la mitad recibe una pensión jubilar no contributiva pequeña y el resto no tiene pensión jubilar. Dado que no existe suficiente oferta de espacios de cuidado y atención al adulto mayor más que para los abandonados, o las escasas iniciativas privadas pagadas, la pensión jubilar es un ingreso clave para apoyar, en lo que sea posible, una vida digna e independiente de los adultos mayores.

El Estado ecuatoriano, como muchos de los Estados de la región, es un Estado de bienestar en ciernes, con varios desafíos para enfrentar los problemas de pobreza y desigualdad. La heterogeneidad del mercado laboral, con un sector informal abultado y muy relacionado con pobreza y baja productividad, hace que los factores de movilización social clásicos no operen del mismo modo que en otros contextos, como el europeo. Estos problemas estructurales del mercado laboral están detrás de la baja cobertura de la seguridad social y de las restricciones en los sistemas de protección social. La política de protección y seguridad social es una necesidad para todos y particularmente para la población excluida que tiene muy pocas posibilidades alternativas de protección en el sector privado, o en las redes familiares y comunitarias para enfrentar contingencias, menos aún durante la vejez.

En Ecuador se ha avanzado mucho en establecer normas y protocolos de seguimiento y exigencia de cumplimiento para la afiliación a la seguridad social, que ha dado sus frutos en 
el incremento notable de la cobertura desde el 2007. Sin embargo, la posibilidad de incorporar a más afiliados enfrenta la realidad de sectores económicos de baja productividad, o grupos poblacionales excluidos del mercado laboral con pocos recursos para enfrentar los costos de afiliación. El Estado ha venido subsidiando anualmente un $40 \%$ de las pensiones jubilares del IESS, y un 60\% de aquellas del ISSFA e ISSPOL, estructura de financiamiento regresiva en el gran contexto de la sociedad ecuatoriana, porque subsidia mucho más a la población afiliada que a aquella que carece de un seguro social. Las pensiones jubilares no contributivas compensaron en parte esta deficiencia, sin embargo, no equilibraron la balanza en la estructura de subsidios. El retiro del subsidio del $40 \%$ de las pensiones y la disposición de usar el subsidio solo cuando éste sea necesario, deja, por otra parte, pendiente la necesidad de pensar fuentes de financiamiento sostenibles en el tiempo para el pago de pensiones que, ineludiblemente, irá creciendo en el tiempo.

De este modo, el sistema de protección y seguridad social enfrenta una insuficiencia más estructural en la cobertura y la necesidad de pensar más sosteniblemente el financiamiento de una expansión de la misma para sectores que requerirán más apoyo de recursos públicos (sector informal, mujeres y jóvenes). Por otro lado, el sistema enfrenta una coyuntura económica adversa, que conduce a tratar el tema del financiamiento y la corresponsabilidad entre los trabajadores, los empleadores, la unidad económica familiar, y el Estado con mucho cuidado.

El problema del financiamiento, además, enfrenta un contexto donde se busca no solo universalizar la pensión jubilar, sino también la educación y la atención de salud. Por tanto, cualquier solución debe considerar estos múltiples objetivos que demandan ingentes cantidades de recursos y observar los criterios de equidad, solidaridad, suficiencia, progresividad y sostenibilidad. Ello implica la necesaria construcción de pactos de largo plazo entre los actores de interés sociales y políticos, donde se incluyan no solo a los grupos organizados afiliados a la seguridad social, sino a los grupos que no cuentan con seguridad social. El pacto se ha construido constitucionalmente por el lado de los beneficios, pero no por el lado del financiamiento, aquí todavía queda un largo camino a recorrer.

Los siguientes criterios buscan impulsar un sistema de pensiones que garantice opciones económicas y sociales para un envejecimiento digno e impida el empobrecimiento de la población en esa etapa de la vida. Gran parte de estos criterios están siendo discutidos en Ecuador, algunos ya han sido aprobados legalmente y otros son parte de los análisis de los actores institucionales y políticos.

Existen al menos cuatro requisitos claves para cualquier reforma a la seguridad social: 1) Una visión clara e integral del sistema de pensiones, dentro de un sistema de protección social universal que incluya tanto el análisis de la evolución fragmentada de la seguridad social - como en el caso ecuatoriano - como el dinamismo y las complejidades del mercado laboral. Por tanto, es importante e ineludible construir una visión sistémica. 2) Una arquitectura institucional de la seguridad social sólida y coherente. Por una parte, preparada para una gestión adecuada que tiene que adaptarse a los cambios demográficos y del mercado laboral y ser capaz de gestionar estrategias innovadoras de afiliación y prestaciones. Por otra parte, preparada para la coordinación de acciones entre la seguridad social y la protección social no contributiva, producto de una planificación integral e integradora de todo el sistema de protección 
social. 3) Financiamiento sostenible, basado en principios de solidaridad y progresividad, que requiere la corresponsabilidad entre trabajadores y empleadores, pero también del Estado, las familias y la sociedad en su conjunto. La factibilidad de ello requiere de la construcción de un pacto social de largo plazo que vaya más allá de la buena voluntad de la universalización de la seguridad social y llegue a la contribución efectiva y justa de los actores de interés. 4) Alta voluntad, liderazgo y legitimidad política de las autoridades de gobierno, que deberán soportarse en acuerdos políticos y sociales para apoyar el financiamiento, la progresividad del subsidio público, el soporte al empleo digno y a la afiliación a la seguridad social.

La complejidad de una reforma orientada a la universalización de las pensiones jubilares que parte de una situación de desigualdad aguda, requiere de acciones inmediatas y de acciones estructurales que implican plazos más largos:

Acciones inmediatas: i) La pensión jubilar no contributiva es muy importante en la transición hacia una mayor cobertura de la seguridad social, y hay que extenderla progresivamente en función de los recursos disponibles, empezando por los más pobres. Para ello, es importante redirigir subsidios hacia quienes más lo necesitan; es decir, para aquellos que han permanecido al margen de la seguridad social porque no han podido generar ingresos o sus ingresos han sido muy bajos. Estas pensiones no contributivas deberán ir disminuyendo en el tiempo, conforme se vayan enfrentando y resolviendo los problemas del mercado laboral y de la afiliación. ii) Adicionalmente, la provisión de servicios de salud progresivamente gratuitos por parte del Estado es clave para evitar el rápido deterioro de la población envejecida, pues la pensión jubilar solo es un soporte básico de ingresos, insuficiente para las necesidades de cuidados de la salud. En el caso ecuatoriano, se debe consolidar la red pública integral de salud con una adecuada coordinación de las prestaciones de salud de los distintos institutos de la seguridad social y del Ministerio de Salud Pública. Si bien se ha avanzado en esta coordinación, restan todavía desafíos muy grandes, tanto en términos de manejo presupuestal, como de funcionamiento y articulación real.

Acciones estructurales. Ampliar la cobertura de la seguridad social es el tema de fondo para mejorar las condiciones de protección de la ciudadanía y asegurar en el largo plazo una pensión jubilar adecuada para la población en edad avanzada. Es importante incorporar a los grupos excluidos de la seguridad social a la afiliación, sobre todo aquellos grupos que se encuentran en situación de mayor vulnerabilidad, que no tienen alternativas de protección como son los siguientes: i) Trabajadores independientes del sector informal. Reconociendo que el gran volumen de trabajadores independientes está en el sector informal y que existen restricciones objetivas a la cotización a la seguridad social por su bajo nivel de ingresos, el Estado debe reorientar sus subsidios para apoyar parcialmente la cotización bajo un nivel definido de ingresos - en función del salario básico unificado- y promover cotizaciones de los trabajadores de acuerdo a su capacidad de pago. Esto no es del todo nuevo en Ecuador, el Seguro Social Campesino lo hace en el sector rural para campesinos sin relación laboral, pero es prudente adecuarlo de mejor manera para mejorar los beneficios que básicamente se concentran en pensiones y salud. La idea es extender esta lógica al nivel urbano donde se tiene una gran proporción de trabajadores independientes, en gran parte informales, que debería ingresar progresivamente al sistema de seguridad social con cotizaciones parcialmente subsidiadas. Será 
menos costoso subsidiar la cotización actual que, al final, pensiones jubilares no contributivas. ii) Trabajadores independientes formales e informales. Se deben considerar mecanismos más flexibles para el pago de cotizaciones y recaudación, que se adecúen a su tipo de trabajo, ritmos y frecuencia de generación de ingresos. Los pagos podrían ser permanentes o eventuales según la estabilidad o la estacionalidad del ingreso. iii) Personas no remuneradas a cargo de las tareas de cuidado en el hogar, que normalmente son mujeres. En este caso, Ecuador ya dio un paso con la apertura de la opción de afiliación de este grupo poblacional y la instauración de una contribución corresponsable entre la unidad económica familiar y el Estado para el caso de ingresos menores al 150\% del salario básico unificado. Los beneficios cubren pensiones jubilares, de invalidez, mortuoria, viudedad y orfandad.

La afiliación en estos grupos puede ser voluntaria y captar progresivamente una mayor proporción de la población. El impulso a la afiliación de estos grupos debe ir acompañada de una activa estrategia de comunicación, información y capacitación para que la población conozca las ventajas de contar con un sistema de seguridad social y su protección durante el ciclo de vida. Dicha estrategia debe prestar particular atención a los jóvenes.

El cuidado de la salud es muy importante y requiere de políticas de salud que apoyen un envejecimiento en mejores condiciones (Buen Vivir) y una red pública integral de salud fortalecida y consolidada (operadores del Ministerio de Salud Pública y de la seguridad social) que, como manda la Constitución de Ecuador 2008, garanticen la promoción, prevención, curación, rehabilitación y atención integral en salud y fomenten prácticas saludables en todos los ámbitos de la vida. Por supuesto, los temas de financiamiento son claves y ameritan por un lado una estrategia bien pensada de progresividad de la gratuidad de las prestaciones, por otro, una orientación hacia las actividades preventivas para reducir los costos y minimizar la enfermedad, y finalmente un pacto social que redunde en un pacto fiscal para asignar los recursos posibles.

Finalmente, las políticas laborales y económicas. Se requiere de políticas laborales que impulsen el empleo y el salario dignos, con un sistema de protección social adecuado, aspecto que en Ecuador ha tenido un gran avance. Sin embargo, quedan por resolver los problemas del sector informal que tienen que ver con la supervivencia de sectores excluidos del mercado laboral formal que gestionan sus propios emprendimientos en condiciones de muy baja productividad, que a su vez redunda en bajos ingresos y poca capacidad de cotización. En este campo, las políticas económicas más inclusivas y orientadas a dinamizar sectores como las PYMEs, que generan empleo formal, y las políticas sectoriales para fortalecer a los pequeños productores campesinos y a la llamada economía popular y solidaria en Ecuador son claves, y son las que en el largo plazo pueden ir resolviendo los problemas estructurales del empleo, junto a las políticas sociales de fortalecimiento de capacidades.

Si se resuelven en el tiempo los profundos problemas estructurales de la población, sobre todo en el mercado laboral, en casos como el ecuatoriano, la necesidad de asistencia social para la protección en la vejez se irá reduciendo en el tiempo. Es importante, sin embargo, en la transición dar una protección mínima a quienes están desprovistos de una pensión jubilar y asistir las cotizaciones de quienes no pueden cubrirla totalmente en su fase laboral activa.

En suma, el esfuerzo por construir un sistema de protección social más integrado para los adultos mayores, en la parte correspondiente a pensiones, requiere de una estrategia integral 
de corto, mediano y largo plazo. Ecuador enfrenta este esfuerzo frente a ineludibles restricciones presupuestarias y un ciclo económico desfavorable, que obligan a pensar en el financiamiento y la sostenibilidad dinámica de un sistema que proteja derechos básicos de la población. Es necesario concentrar los subsidios en quienes más lo necesitan, sobre todo en los excluidos de la seguridad social. El país ya ha dado varios pasos para reformar la seguridad social, algunas decisiones se han tomado y otras están en estudio, pero siempre conviene reflexionar sobre ciertos criterios para esa necesaria reforma - desde la realidad ecuatorianaque pueda aportar a la construcción de sistemas de seguridad social para países con parecido desarrollo relativo y similares características sociodemográficas en la región.

\section{REFERENCIAS}

Banco Mundial. (2012). Ecuador, Las Caras de la Informalidad. Washington DC, USA: Banco Mundial. Cecchini, S. y Martínez, E. (2011). Protección social inclusiva en América Latina. Una mirada integral, un enfoque de derechos. Santiago de Chile, Chile: CePal.

CEPAl (2010). Panorama Social de América Latina 2009. Santiago de Chile, Chile: CEPAL.

Cepal (2014a). Panorama Social de América Latina 2013. Santiago de Chile, Chile: Cepal.

CEPAL (2014b). 20 años de transformaciones hacia la igualdad. Recuperado de http://www.cepal.org/noticias/paginas/8/33638/141118_Beijing_20_AliciaBarcena.pdf

CEPAL (2014c). Panorama Económico y Social de la comunidad de Estados Latinoamericanos y Caribeños 2014. Santiago de Chile, Chile: CEPAL.

CEPAL (2014d). Observatorio Demográfico 2013. Santiago de Chile, Chile: CEPAL.

CePal (2015). Balance Preliminar de las Economía de América Latina y el Caribe 2015. Santiago de Chile, Chile: CEPAL

Durán, F. (2008). Diagnóstico del Sistema de Seguridad Social del Ecuador. Lima, Perú: OIT.

IESS (2015). Sostenibilidad de la Seguridad Social, implementación de medidas para mejorar la eficiencia en el gasto. (Presentación no publicada).

IEss (28 de julio de 2015). Resolución del Consejo Directivo del IEss. Quito, Ecuador.

INEC (2015a). Proyecciones Poblacionales. Recuperado de http://www. ecuadorencifras.gob.ec/ proyecciones-poblacionales/

INEC (2015b). Encuesta Nacional de empleo, desempleo y subempleo. Indicadores laborales, Diciembre 2015. Recuperado de http://www.ecuadorencifras.gob.ec/documentos/web-inec/EMPLEO/2015/ Diciembre-2015/Presentacion_Empleo_dic_15.pdf

Ley Orgánica para la Justicia Laboral y el Reconocimiento del Trabajo en el Hogar. (20 de abril de 2015). Registro Oficial, 2(483), (s. n.)

Maldonado, C. y Palma, A. (2013). La construcción de pactos y consensos en materia de política social. Apuntes para un marco de análisis». Serie Políticas sociales, (179). Santiago de Chile, Chile: CEPAL. Mesa-Lago, C. (2004). Las reformas de Pensiones en América Latina y su impacto en los principios de la Seguridad Social. Serie Financiamiento del desarrollo, (144). Santiago de Chile, Chile: CEPAL. MCDS(2009). Agenda Social 2009-2011. Quito, Ecuador: MCDS.

MCDS (2012). Agenda Social 2012-2014. Quito, Ecuador: MCDS. MCDS(2014a). Agenda Social al 2017. Quito, Ecuador: MCDS. 
MCDS(2014b). Propuesta de políticas para adultos mayores (Presentación). Quito, Ecuador: MCDS.

MCDS(2014c). Diagnóstico de la Seguridad Social (Presentación). Quito, Ecuador: MCDS.

Naranjo, M. (2013). Sistemas de Protección Social en América Latina y el Caribe: Ecuador. Santiago de Chile, Cile: CEPAL.

OIT (2012). Panorama Laboral 2012. Lima, Perú: OIт.

Osorio, C. (2014). La difusión de Programas de Transferencia Condicionada en América Latina 1990-2010. (Tesis doctoral). Universidad Autónoma de Barcelona, Barcelona, España.

Robles, C. (2012). La Protección social contributiva en América Latina: una revisión de sus avances y desafíos. En Konrad Adenauer Stiftung y Universidad Rafael Landívar 2012, $5^{\circ}$ Seminario Internacional de Política Social: La Agenda social para el desarrollo de Guatemala. Ciudad de Guatemala, Guatemala.

Sánchez, J. (2012). Equidad y Políticas Sociales: algunas reflexiones para el caso ecuatoriano. En A. Serrano (Coord.), ¡A (re)distribuir! Ecuador para todos. Quito, Ecuador: SENPLADES.

SEnPlades (Secretaría Nacional de Planificación y Desarrollo). (2014). Estrategia Nacional para la Igualdad y la Erradicación de la Pobreza. Quito, Ecuador: SENPLADES. 
